\title{
Method of Intelligent Plan Recognition
}

\author{
Zhang Lin ${ }^{\mathrm{a}^{*}}$, Liu Huayun,Cheng Weiming \\ The 28th Research Institute of China Electronics Technology Group Corporation, Nanjing 210007 , \\ China \\ achen7225@hotmail.com
}

Keywords: plan recognizer, intelligence, intelligent plan, plan recognition

Abstract. Plan recognition as an important research direction of artificial intelligence, are widely used in many aspects of the intelligent system. This article from the concept, classification, methods, research situation and application field, introduces the research of plan recognition. In this article, provide a certain theoretical basis for the further research plan recognition.

\section{Introduction}

Theory of plan recognition is a very active in the study of artificial intelligence research direction, has been widely used in natural language understanding, knowledge and understanding of calculus and other computer system in many ways. Plan recognition [1] is based on the observations to estimate target is execution plans. These plans are usually under the assumption that the recognizer has complete knowledge, and the target under the condition of no error.

Early plan recognition system is mainly used in the story to understand and expert system, but now plans to identify application extension to the operating system, natural language understanding, the military defense system, medical diagnosis system, network intrusion detection system and program understanding and automatic driving and other areas.

So far, in the world, although the current plan recognition research has begun, but have no plans to shape recognition system, recognition technology research project is just at the primary stage.

\section{The Concept of plan recognition}

A. intelligent plan recognition

From the concept of intelligent plan recognition understanding, can put the smart plan recognition process understanding in the process of extracting effective planning process. Planning is a process of the method to solve the problem, planning system is a system to solve the problem, can through a series of actions, in order to perform this action sequence to perform specific tasks, and ultimately achieve a particular goal. Is on the contrary, in this paper, we study the smart plan identification process plan recognition is to point to by observing object to perform a series of parts, trivial, dispersed operations to infer the object's target and execute the plan process, and predict future action sequences.

B. plan recognition

Plan recognizer is a software application, by observing a goal in the first few, a few scattered or observed target action, to identify the target program is executing. Plan recognition input is observed and problems in the field of background knowledge, the output is to be able to explain the observed plan hypothesis [2].

C. aggressive planning

Aggressive behavior [3], is defined by the Agent performs certain aggressive and destructive nature of instantiation operation, it may be partial or fragmentary, also may be immediate or real-time. However, these movements of the specific objectives are intended to destroy rivals Agent system (software, hardware or software system, hardware system), so as to achieve a malicious Agent to achieve on the target system for the purpose of some instantiation operation. an entity in initial state after executing a series of aggressive behavior, such as act 1 , act $2 \ldots \mathrm{N}$, behavior, and eventually 
reached the target state, then, this a series of movements or the sequence of action behavior of $1,2, \ldots$, $\mathrm{N}$, is called an attack plan.

\section{The classification of plan recognition}

A. The classification according to the role of the agent

Intelligent plan recognition is based on the inference Agent in plan recognition in rats can be roughly divided into the following two categories[1]:

- Hole type plan recognition: this method of identification in the process of the recognition, observation and reasoning by Agent did not affect the plan recognition process, through the observation of attract sb.'s attention to infer Agent target.

- Intentional plan recognition: this method of identification in the process of recognition, to be observed and reasoning Agent effects plan recognition process and this effect can be divided into two kinds: help or hinder the recognition process. In this environment when Agent realized or find others in observation and reasoning about their plans, Agent will take effective measures to block the plan recognition process.

B. The classification according to rule base

Intelligent plan recognition according to whether a rule base can be divided into[1]:

- Plan recognition of having rules: using hierarchical task network layer, events, knowledge map or other means to describe the planning, and it based on these plan recognition.

- Plan recognition of no rules: the plan recognizer does not need according to the preset program can be given recognition results. No rule library plan recognition through must have specific rules to plan recognition restrictions.

\section{The Method}

A. default reasoning

Default theory is in the knowledge under the condition of incomplete making reasoning can continue a no monotonic reasoning. Default reasoning is the core of the default or assuming certain proposition was established under the premise of reasoning. Carberry [4J according to natural conversation analysis as well as to human reasoning in psychological research presents a plan recognition model based on default reasoning. Its main idea is supported through appropriate default reasoning is used to delay the groundless conclusion until further evidence appears, thus gradually update user programming system model.

B. the limit theory

Limit theory is a nonmonotonic reasoning method, but also to study the earliest nonmonotonic reasoning method, is the McCarthy[5] in the nineteen seventies end puts forward, he did not introduce any new operator or logical symbols, only in the classical logic framework for representation of non monotonic special reasoning form. Limit theory is thecore idea: if a sentence describes a proposition, then it describes only the proposition, not expansion and extension. For example, if the boat to cross the river, then it means that the only ship to cross the river, any other possible tools to cross the river can be considered.

Human and intelligent computer often need to conclude some conclusions: with certain properties or relations among objects are only meet these relationships to objects. McCarthy limit theory formalized this reasoning. As a result of limit theory in first-order logic to add some rules, therefore, can be used with conventional logic to formal nonmonotonic logic. But the plan recognition problem is usually a nonmonotonic logic reasoning problems, thus may limit theory and plan recognition problem of combining.

Kautz plan recognition problem is solving the observed movements minimal planning, which is very similar to limit theory. But because the limit theory contains two order logic, calculation is complex, therefore, Kautz is based on limit theory put forward three hypotheses (exhaustiveness 
assumption, exclusive hypotheses, using component hypothesis), and not directly defined approach to solving the plan recognition problem. In 2002, Yunfei Jiang and Ning Ma [6J in the Kautz plan recognition based on the combination of the above two methods. The plan recognition problem solves the method. According to Kautzplan recognition, Yunfei Jiang, Ning Ma gives a decomposition and enumeration of the concepts, and gives the solving plan recognition algorithm for the problem of limit. According to define and plan recognition, they give theorem, to illustrate the observed phenomenon do the limit obtained solution set and the observed phenomenon of the minimal planning set is the same. Yunfei Jiang dependsKautz on the plan recognition method, first proposed the theory with limited direct solving plan recognition problem, and make up the inadequacy of Kautz plan recognition, enhance the fault tolerant ability of plan recognition.

C. Bayesian network

Chamiak and Goldman [7] proposed the plan recognition based on Bayesiannetwork model. They will plan recognition as a special case of reasoning, and describe a movement based on some observed to generate possible explanations for the planning graph recognition method. The main problem of this approach is that when the knowledge base and evidence collection increases, the size of the Bayesian network will rapidly increase, so does not apply to more complex problem areas.

D. syntax analysis

In 1990 Villain [8J based on the Kautz theory as syntax analysis of plan recognition theory. He did not actually uses the syntax analysis method for handling the plan recognition problem, but through the reduction of plan recognition limit to syntax analysis, to study the theory of Kautz complexity. The grammar analysis model of plan recognition is mainly used in the story comprehension and conversation analysis.

E. goal graph

The usual plan recognition is established on the basis of the plan base [9]. In 2000, Jun Hong from the plan get inspiration, put forward a kind of need not plan base of target identification methods, namely the target of plan recognition method. Its main idea is to construct a target image, and then through the goal graph analysis for plan recognition. This method of identification feature is that it does not require planning library, but first constructs a target graph, then analyzes the target image target recognition and effective planning. Therefore, with the plan library plan recognition, the method has obvious advantages. Because the method broke through must have specific plan library to plan recognition limits, able to make new plan recognition, so it is suitable for hostile plan[10] [11] and other intelligentbody in a hostile state [12] [13] identification. But it is still not perfect, can only be explained by past actions and cannot predict future actions, therefore, the method is suitable for application in story comprehension, game[14], a software consulting system, database query optimization and customer data mining and other fields.

\section{Conclusion}

The branch of artificial intelligence, intelligent planning and plan recognition has become an important area of research. From the direction of the intelligent identification system planning, this paper introduces the concept, classification, methods and systems at home and abroad research situation and application of the artificial intelligence planning field. It is not difficult to see, artificial intelligence research plan recognition technology will be launched, and will become the mainstream of the research direction of artificial intelligence, more and more attention of scholars and experts.

\section{References}

[I] R.X Liu, WX.Gu, M.B. Yin, "Intelligent plan recognition and its application", Computer engineering.

[2] XM. Ji, YF Lian, XL. Xu, We.Jia, "Research and development of intrusion detection technology", Computer Simulation, 2004.Vol. 2I.No.II.11. 
[3] WX. Gu, Lei Wang, YL. Li, "Research for adversarial planning recognition and reply in the complex domains and the more agent's conditions". Machine Learning and Cybernetics, 2005:225-230.

[4] Sandra Carberry. "Incorpating Default Inferences into Plan Recognitin".In Proceedings of the Eighth National Conference on Artificial Intelligence, Bostom, Massachusetts, 1990471-478. [5] McCarthy J. "Circumscription-A form of non-monotonic reasoning" [J]. Artificial Intelligence, 1980,1327-39.

[6] YF Jiang, Ning Ma, "Based on the defined plan recognition problem solving", Chinese Journal of computers, 2002 (25), 1411-1416.

[7] Charniak, E, Goldman, R. P. A "Bayesian model of plan recognition". Artificial Intelligence, 1993, 64(1) 53-79.

[8] Villain, M. Getting serious about parsing plans "a grammatical analysis of plan recognition". In Proceeding of the 8th National Conference on Artificial Intelligence. Boston: AAAI Press. 1990: 190197.

[9] E.D. Sacerdoti. "A Structure for Plans and Behaviour".Elsevier, 1977.

[10] Steven Willmott, Alan Bundy, John Levine, Julian Richardson. "Adversarial Planning in Complex Domains".Submitted to ECAI 98 January 20, 1998.

[II] JaimeG. Carbonell, Counterplanning "A Strategy-Based Model of Adversarial Planning in Real-World Situations".Artificial Intelligence 16 295-329, Computer Science Department, Carnegie-Mellon University, U.S.A, 1981.

[12] Steven Willmott, Julian Richardson, Alan Bundy, and John Levine. "An Adversarial Planning Approach to Go". Submitter to ECAI, 1997. [13] e. Applegate. e. Elsaesser. and D. Sanborn. "An architecture for adversarial planning". IEEE Transactions on Systems, Man and Cybernetics, 20(1), 1990: 186-294.

[14] S. Willmott. "Adversarial Planning and the Game of Go". Master's thesis, Department of A 